\title{
IPBES para ciudadanos: breve aproximación a la Plataforma Intergubernamental Científico- Normativa sobre Biodiversidad y Servicios Ecosistémicos
}

\section{IPBES for Citizens: A Brief Approach to the Intergovernmental Science-Policy Platform on Biodiversity and Ecosystem Servicesu}

\section{Albaluz Ramos Franco ${ }^{\text {ab }}$ y Leyde Katerine Cabrera Ruíz ${ }^{\mathrm{ac}}$}

\author{
ab Grupo de investigación Biología para la conservación, Facultad Ciencias Básicas, Universidad Pedagógica y Tecnológica de \\ Colombia, Colombia \\ balbaluz.ramos@uptc.edu.co http://orcid.org/0000-0001-6432-4061 \\ cleyde.cabrera@uptc.edu.co https://orcid.org/0000-0003-2223-6877
}

\section{RESUMEN}

La Plataforma Intergubernamental Científico-Normativa sobre Biodiversidad y Servicios Ecosistémicos (IPBES) es un órgano multilateral de la Organización de las Naciones Unidas que busca involucrar el conocimiento científico en las decisiones políticas de los Estados Parte. La IPBES está constituida por cinco secciones no jerarquizadas que tienen la tarea de reunir y evaluar toda la información mundial sobre los temas priorizados para elaborar una serie de informes (Ilamados Evaluaciones), los cuales serán la principal herramienta de los estados para la creación de políticas, normas y programas en pro de la conservación de la biodiversidad. Las Evaluaciones hechas hasta el momento abarcan los temas de polinización y polinizadores, escenarios y modelos de biodiversidad y servicios ecosistémicos, degradación y restauración de la tierra, evaluación regional de biodiversidad y servicios ecosistémicos para cada continente y la Evaluación Global de biodiversidad y servicios ecosistémicos. A partir de

Citation: Ramos Franco, A., y Cabrera Ruiz. L. K. (2020). IPBEs para ciudadanos: breve aproximación a la Plataforma Intergubernamental Científco-Normativa sobre Biodiversidad y Servicios Ecosistémicos. Mutis, 10(2), 37-50. https://doi.org/10.21789/22561498.1616

Recibido: Junio 1, 2020. Aceptado: Agosto 20, 2020.

Copyright: $\odot 2020$. Ramos Franco, A., y Cabrera Ruiz. L. K. (2020). This is an open-access article, which permits unrestricted use, distributions and reproduction in any medium, provided the original author and source are credited.

Competing Interests: The authors have no conflict of interest. sus resultados, se presentan algunas reflexiones sobre las acciones que la sociedad, la comunidad científica y los tomadores de decisiones pueden ejercer para contribuir a la conservación de la biodiversidad y sus servicios ecosistémicos.

Palabras clave: ONU, tomadores de decisiones, Evaluaciones, biodiversidad. 


\section{ABSTRACT}

The Intergovernmental Science-Policy Platform on Biodiversity and Ecosystem Services (IPBES) is a multilateral body of the United Nations that seeks to involve scientific knowledge in the political decisions by State Parties. The IPBES is made up of five non-hierarchical sections, which have the task of gathering and evaluating all the global information on prioritized topics in order to prepare reports (called Assessments) that will be the main tool of the states for the creation of policies, regulations, and programs aimed at the conservation of biodiversity. Assessments published so far encompass the issues of pollination and pollinators, scenarios and models of biodiversity and ecosystem services, land degradation and restoration, regional assessment of biodiversity and ecosystem services for each continent, and the Global Assessment of Biodiversity and Ecosystem Services. Based on these assessments, recommendations are made on how society, the scientific community and decision-makers can contribute to the conservation of biodiversity and ecosystem services.

Keywords: UN, decision makers, Assessments, biodiversity.

\section{INTRODUCCIÓN}

El Convenio de Diversidad Biológica (en adelante CDB) es un tratado internacional que se abrió para firma el 5 de junio de 1992 en la Cumbre de la Tierra en Río de Janeiro (ONU, 1992). Este tratado, ratificado por 196 estados (en adelante "las Partes"), tiene como objetivo la conservación de la diversidad biológica, la utilización de sus componentes y la participación justa y equitativa de los beneficios derivados de la utilización de los recursos genéticos. Este instrumento, netamente gubernamental, requirió desde su inicio la asesoría de expertos en la materia, por lo que establece en su Artículo 25 el Órgano Subsidiario de Asesoramiento Científico, Técnico y Tecnológico (SBSTTA, por sus siglas en inglés). Desde 1994, fecha de entrada en vigor del convenio, hasta la actualidad, el SBSTTA se ha reunido en 22 oportunidades, en las que, de acuerdo con su mandato, produce "Recomendaciones" para consideración de la Conferencia de las Partes, máximo órgano decisorio del CDB que se reúne cada dos años.

Sin embargo, en 2008, la comunidad científica, en particular Laikre et al. (2008), alertó sobre la pérdida de objetividad y credibilidad científica de la información que se estaba utilizando en el Órgano. Con el fin de responder a la lluvia de críticas, las Partes iniciaron en 2008 un proceso de negociación en el que destacó el liderazgo de Colombia, el cual finalizó en el 2012 con la creación de la Plataforma Intergubernamental Científico-Normativa sobre Biodiversidad y Servicios Ecosistémicos, un nuevo órgano que centraría sus decisiones en la más robusta recopilación de informa- ción científica a nivel mundial. En 2014 se hace oficial la cooperación entre IPBES y CDB a través de un memorando que establece la sinergia y la no duplicación de funciones y decisiones (Memorando, 2014). A la fecha, IPBES cuenta con 137 estados miembro (IPBES, 2019).

Existe un sinnúmero de publicaciones internacionales que relatan el qué, por qué y para qué de IPBES, incluyendo fuertes críticas a su base conceptual (Braat, 2018; Díaz et al., 2015; Díaz et al., 2018; Faith, 2018; Kenter, 2018; Pascual, 2017; Peterson et al., 2018). Sin embargo, quienes trabajan diariamente en asuntos de biodiversidad y conservación en Latinoamérica y Colombia, los entes territoriales locales, e incluso el ciudadano común, no están familiarizados con esta importante asamblea; probablemente debido a la condición netamente gubernamental que la soporta y la barrera idiomática que predomina, dado que sus discusiones son en inglés. Por lo anterior, este trabajo pretende brindar información básica sobre la IPBES en un lenguaje que cualquier persona interesada pueda entender, discerniendo la importancia de las decisiones que se toman al interior de éste organismo.

\section{OBJETIVOS Y ESTRUCTURA DE IPBES}

La Plataforma tiene como fin principal cerrar la brecha existente entre la ciencia y la política, respondiendo las preguntas que los tomadores de decisiones tienen sobre la gestión de la biodiversidad y sus servicios ecosistémicos y acercando el conocimiento que estos necesitan para desarrollar normas o programas que

M U T I S" | Revista electrónica editada por la Facultad de Ciencias Naturales e Ingeniería de UTADEO 
propendan por la conservación de la los mismos, todo ello en búsqueda del bienestar humano y el desarrollo sostenible a largo plazo (Jonsson et al., 2017). Para esto, se formularon cuatro funciones:

- Aceleración de la generación de conocimiento: a diferencia del consenso existente sobre las ciencias atmosféricas que soportan los Acuerdos de Cambio Climático (Brooks, 2014), la pérdida de biodiversidad no tiene un patrón global exclusivo y existen aún vacíos de conocimiento que requieren ser completados para tomar decisiones políticas al respecto.

- Fortalecimiento de capacidades: como consecuencia del punto anterior, la Plataforma debe identificar necesidades de conocimiento y enfocar la cooperación con entes académicos (como la Unión Internacional para la Conservación de la Naturaleza [IUCN, en inglés], el Programa Biodiversidad y Gestión de Áreas Protegidas [BIOPAMA, en inglés] y onU Fellowships Programs) para subsanarlas.

- Evaluaciones: la Plataforma recoge el conocimiento científico, técnico, local e indígena para elaborar documentos Ilamados "Evaluaciones", los cuales tienen un componente científico-técnico fuerte y un resumen de fácil comprensión para tomadores de decisiones; más adelante se explicarán cada una de las Evaluaciones hechas hasta la fecha.

- Soporte para la formulación de políticas: la Plataforma facilita a las Partes herramientas para que los dirigentes políticos puedan legislar normas y programas enfocados en atender los resultados de las Evaluaciones; incluso, para que hagan sus propias Evaluaciones nacionales con mayor nivel de detalle (Álvarez-Dávila et al., 2019).

Una vez comprendido el para qué de la Plataforma, es necesario ver cómo está organizada. En ese sentido, la IPBES está constituida por cinco secciones no jerarquizadas entre sí:

- Plenaria: máximo órgano decisorio en el que participan los delegados de los Estados miembros. Esta asamblea se reúne cada año para ajustar y adoptar los documentos resultado de las Evaluaciones (que se explican más adelante).

- Buró: es el cuerpo administrativo que funciona como una Junta Directiva. Tiene un presidente, cuatro vicepresidentes y cinco oficiales administrativos. Modera las plenarias y participa en la elaboración de los documentos.

- Panel Multidisciplinario de Expertos (MEP): conformado por 25 expertos (cinco por cada una de las cinco regiones de la ONU), quienes son elegidos de entre un grupo de científicos voluntarios que atienden el llamado del buró y sus gobiernos nacionales.

- Secretaría: brazo netamente administrativo que organiza y hace seguimiento a las plenarias y sus decisiones y tiene a su cargo la página web. Tiene su sede permanente en Bonn (Alemania).

- Grupos de soporte: escriben los borradores de los documentos que serán revisados y aprobados en la plenaria, reuniendo el conocimiento existente. Están subdivididos en:
a. Grupos de trabajo
b. Expertos de alcance
c. Expertos para las Evaluaciones específicas

Aunque no se encuentran establecidos como una sección oficial de IPBES, los stakeholders, o grupos interesados, son una parte fundamental de la Plataforma. Desde la primera plenaria (decisión IPBES/1/12 anexo II) se dio vía libre a la participación de ONG, universidades y organizaciones académicas en el marco de las plenarias (IPBES, 2016a; ONU, 2019a). Estos grupos deben solicitar el aval ante el Buró y tienen un día completo de discusión interna previo a la inauguración del evento; allí se reúnen las peticiones o comentarios, que pueden o no ser tenidos en cuenta durante las negociaciones del contenido de las Evaluaciones. A diferencia de los delegados estatales, los stakeholders no tienen voz ni voto durante la plenaria, y ostentan la categoría de observadores.

Desde enero de 2013, IPBES ha desarrollado anualmente sus plenarias, eligiendo siempre un país miembro como anfitrión. Colombia hospedó esta reunión en 2018, en la ciudad de Medellín (Antioquia). 


\section{MARCO CONCEPTUAL}

La elaboración de las Evaluaciones requería de un enfoque estandarizado, que garantizara su replicabilidad y calidad. Por esto, un grupo de 84 científicos, encabezados por Sandra Díaz (Bióloga argentina, Ph.D. en Ciencias Biológicas) y con supervisión del MEP-, elaboró lo que se conoce como el marco conceptual de IPBES, adoptado en diciembre de 2013 (decisión IPBES 2/4) y publicado como artículo científico en 2015 (Díaz et al., 2015), el cual retoma la perspectiva básica de la Evaluación de los Ecosistemas del Milenio sobre los servicios ecosistémicos y le da nuevos valores, incluso un nuevo nombre: contribuciones de la naturaleza a la población (NCP, por sus siglas en inglés). En la figura 1 se aprecian los seis elementos principales del marco conceptual que capturan la complejidad de los retos que se deben superar en aras de lograr una buena calidad de vida para el ser humano en el planeta. De manera general, el esquema del marco conceptual indica la forma en la que los seis elementos interactúan (flechas continuas), cuáles de estas interacciones no son prioridad para la Plataforma (flechas punteadas) y cómo todo esto sucede a través de diferentes escalas temporales y espaciales.

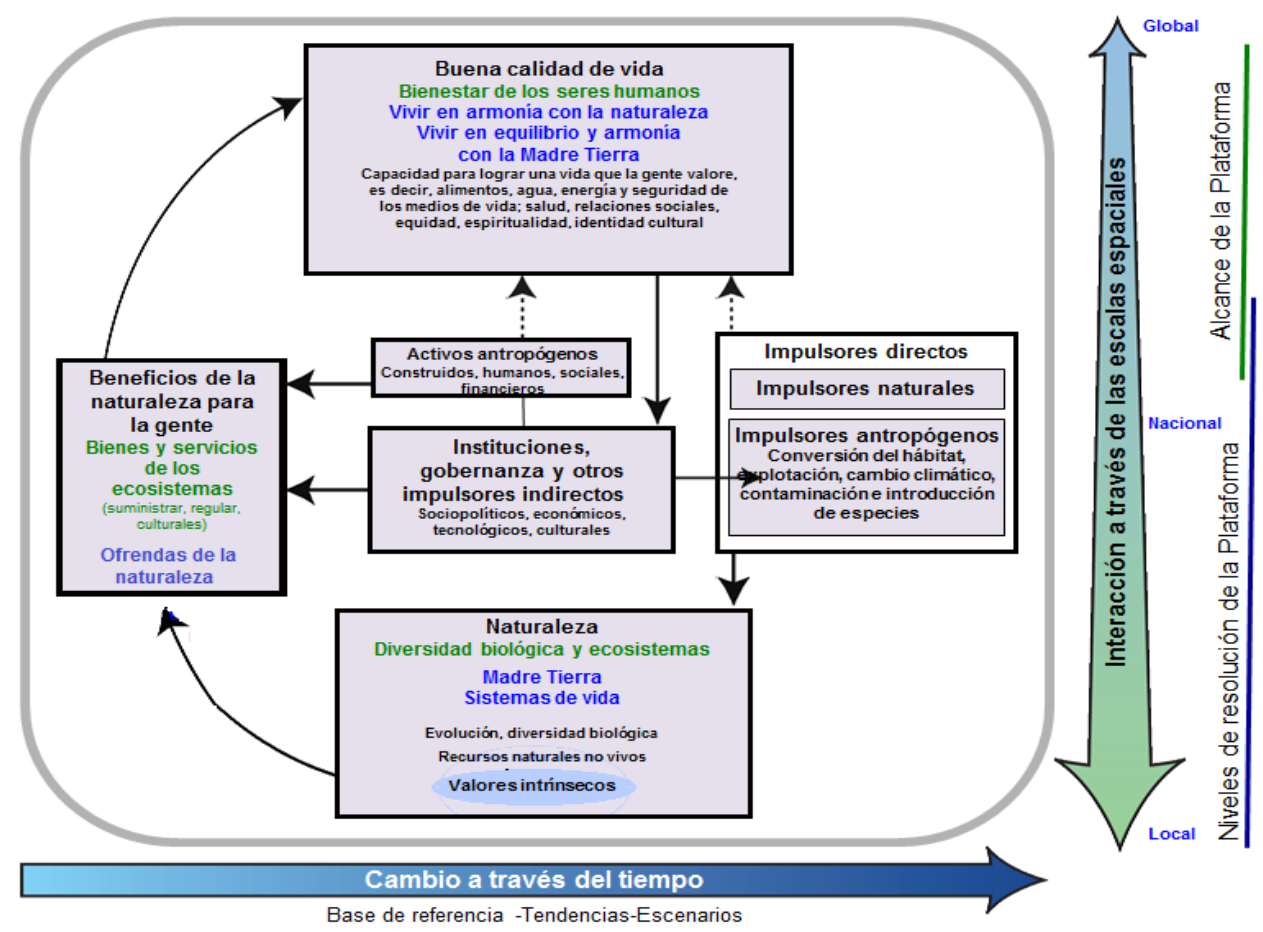

Figura 1. Marco conceptual de IPBES.

Fuente: tomado de la Decisión IPBES 2/4 (ONU, 2013a).

\section{PRIMER PROGRAMA DE TRABAJO}

En la segunda Plenaria, IPBES aprobó el programa de trabajo para el período 2014-2018, en el cual se establecen acciones concretas para el cumplimiento de sus 4 objetivos (figura 2). A partir de este primer plan de trabajo se establecieron metas anuales de cumplimiento, priorizando algunas ante la urgencia de publicación de Evaluaciones específicas (como la de polinización y polinizadores, como se verá más adelante). En el autoexamen realizado en el IPBES-7 se revisó el éxito de cada objetivo, destacando el impacto que han tenido las Evaluaciones publicadas y la aceptación general que los estados han tenido hacia estas; tanto que se seguirá el mismo proceso para los próximos años. Otro logro relevante es el aumento de la producción y difusión del conocimiento a nivel global gracias al programa de jóvenes becarios, quienes participan en todas las actividades de cada una de las Evaluaciones propuestas. La primera cohorte se graduó en el IPBES-6 con la publicación de las Evaluaciones regionales. 
Objetivo de la Plataforma

Fortalecer la interfaz cientifico-normativa de la diversidad biológica y los servicios de los ecosistemas para la conservación y utilización sostenible de la diversidad biológica, el bienestar de los seres humanos a largo plazo y el desarrollo sostenible Funciones, principios operativos y procedimientos de la Plataforma

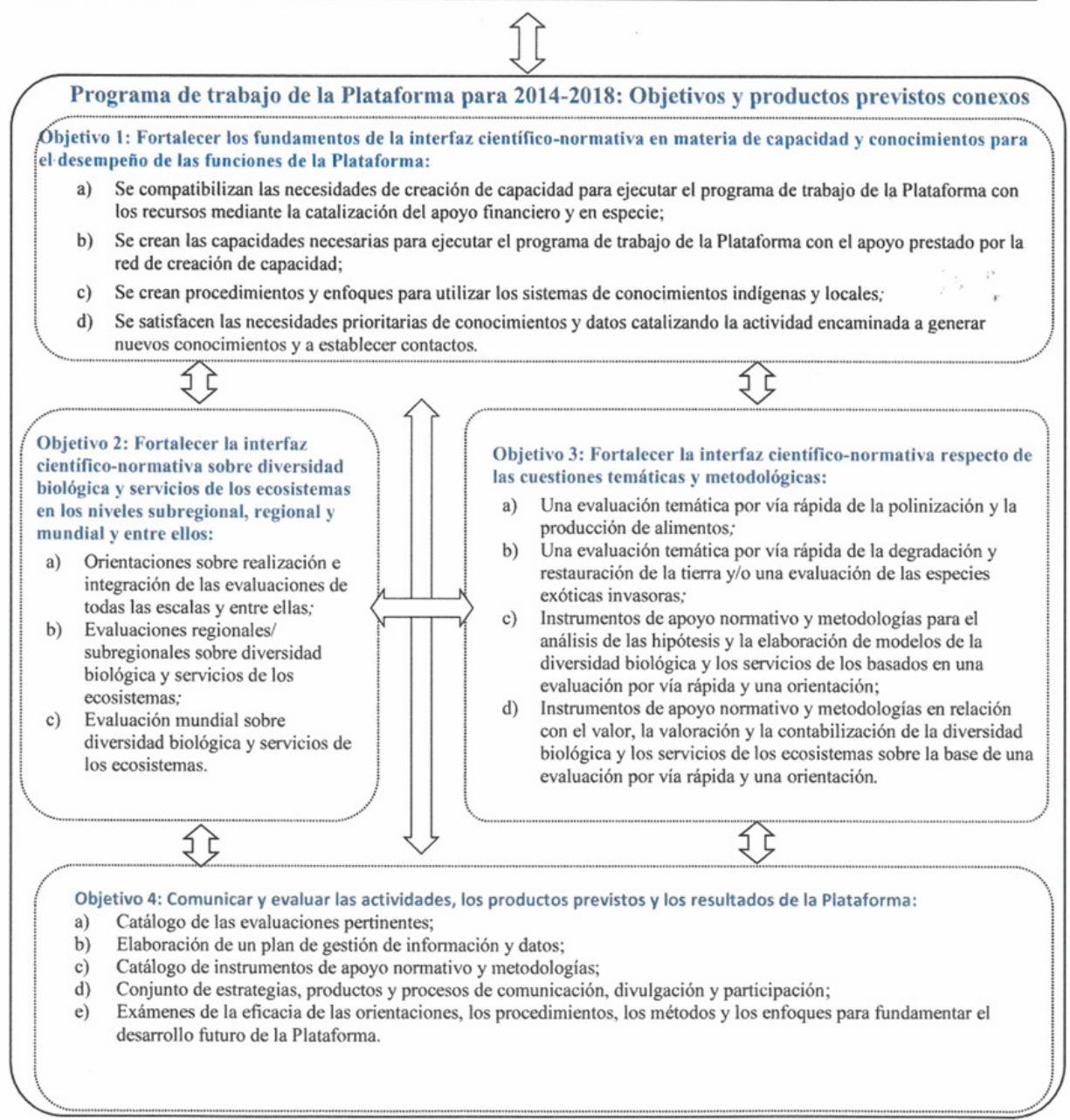

Figura 2. Programa de trabajo 2014-2018.

Fuente: tomado de la Decisión IPBES 2/5 (ONU, 2013b).

En la sesión siete de la Plenaria (decisión IPBES 7/1) se planteó el establecimiento de un nuevo programa de trabajo, con aplicación a 10 años. Algunas de las temáticas a priorizar son:

- Promoción de la biodiversidad para lograr los objetivos de la Agenda 2030 para el desarrollo sostenible (ONU, 2015).

- Causas subyacentes de la pérdida de la diversidad biológica (ONU, 2018a, 2018b).

- Medición del impacto de la industria y las empresas y su dependencia de la diversidad biológica.
Se espera que de allí resulten nuevas evaluaciones temáticas con sus respectivos Resúmenes para Tomadores de Decisiones.

\section{EVALUACIONES}

Luego de unir los comentarios hechos por los grupos de soporte, los stakeholders y el mismo MEP, la Plenaria decide los temas prioritarios a analizar con el fin de presentar un producto escrito: las Evaluaciones. Hasta el momento, existen dos tipologías, una referente a la escala espacial y otra referente a temas específicos. 
Las Evaluaciones contienen dos apartes, a saber: (i) un documento completo dividido en capítulos con todas las especificaciones técnico-científicas del tema o escala analizadas y (ii) un resumen para tomadores de decisiones, en el cual se explican los mensajes principales del primer documento y se hace un breve recuento del conocimiento existente sobre el tema. Este último debe ser discutido por la Plenaria antes de ser aprobado y publicado con el fin de que todas las partes lleguen a un consenso del lenguaje y las posturas políticas que usan sus estados, para que así estas puedan ser aplicadas local y regionalmente.

De manera condensada, se exponen a continuación los temas de la evaluación y sus mensajes principales (figuras 3-7); estos últimos son aseveraciones contundentes y comprobadas con total respaldo científico sobre el presente y futuro del tema en el planeta.

\section{EVALUACIÓN SOBRE LOS POLINIZADORES, POLINIZACIÓN Y PRODUCCIÓN DE ALIMENTOS}

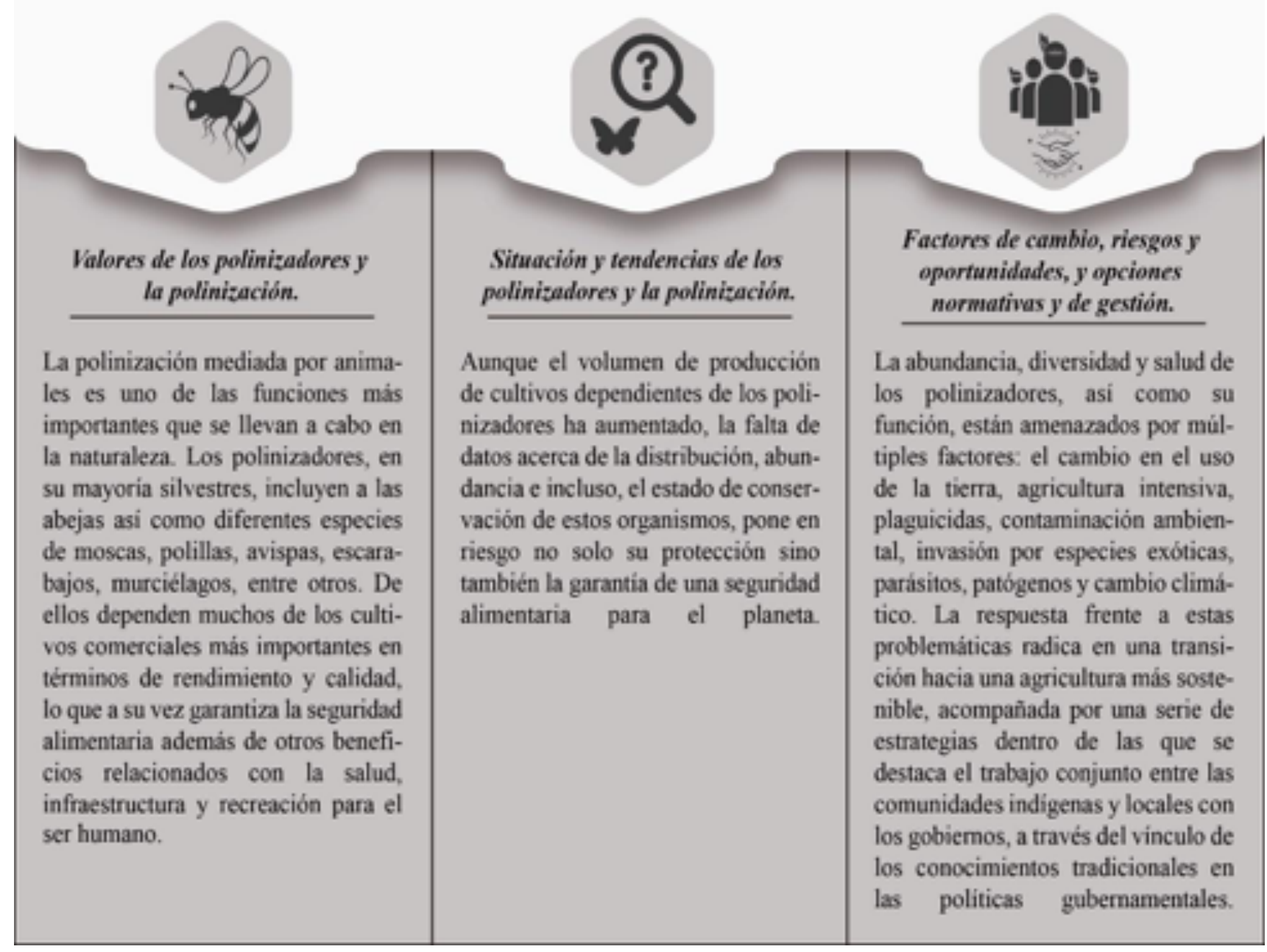

Figura 3. Mensajes principales de la Evaluación sobre polinizadores, polinización y producción de alimentos Fuente: tomado de la Decisión IPBES 4/19 Anexo II (2016). 


\section{Y MODELOS DE LA BIODIVERSIDAD Y SERVICIOS ECOSISTÉMICOS}

\begin{abstract}
Las hipótesis y los modelos pueden contribuir significativamente al apoyo normativo, pese a que varios obstáculos han impedido su uso generalizado.

En el proceso de formulación de politicas y adopción de decisiones, se requiere de herramientas tales como las hipótesis y los modelos, que consisten en aquellas opciones normativas contempladas por los tomadores de decisiones, unidas a sus respectivas consecuencias en la naturaleza, los beneficios que esta da a las personas y la calidad de vida. Dichos mecanismos dependen de la disponibilidad de datos, conocimientos, recursos humanos y técnicos, además de la colaboración y cooperación entre las distintas partes interesadas en el tema.
\end{abstract}

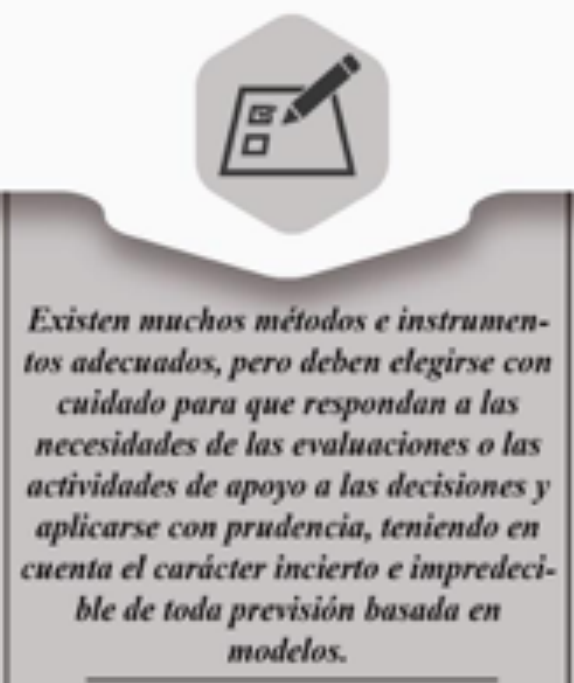

El uso de un enfoque participativo en el que haya un trabajo conjunto entre los responsables de la formulación de politicas, los especialistas y las comunidades indigenas $y$ locales, permite no solo llenar los vacios de información existentes, sino tambièn aplicar $e$ integrar eficazmente las hipótesis y modelos al momento de elaborar politicas adecuadas a cada contexto y escala espaciotemporal pertinente.

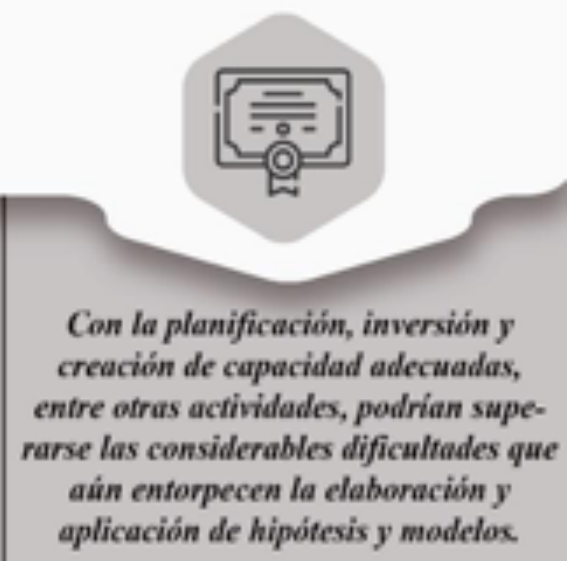

Tanto las hipótesis como los modelos han presentado una serie de inconvenientes persistentes relacionados con su formulación y aplicación apropiadas, teniendo en cuenta los vinculos existentes entre la diversidad biológica, el funcionamiento de los ecosistemas y los servicios ecosistémicos que prestan, así como la relación de estos últimos con la calidad de vida y la integración en los sectores politico, económico, social y ambiental. Esto ha llevado a una deficiencia en la disponibilidad de datos, seguido por la generación de errores de concepto y en consecuencia, una evaluación y adopción de decisiones equivocada, lo que hace necesaria la formación de especialistas y responsables en la formulación de politicas, así como la mejora en el acceso a la información e instrumentos que apoyen esta labor.

Figura 4. Mensajes principales de la Evaluación sobre los escenarios y modelos de la biodiversidad y servicios ecosistémicos Fuente: tomado de la Decisión IPBES 4/19 Anexo IV (2016). 


\section{EVALUACIÓN SOBRE LA DEGRADACIÓN Y RESTAURACIÓN DE LA TIERRA}

\begin{abstract}
La degradación de la tierra es un
fenómeno sistémico generalizado qua tiene lugar en todas las partes del globo terrestre $y$ puede adoptar numerosas formax.

La degradación de la superficie terrestre se ha convertido en una de las consecuencias más importantes derivadas de actividades como la expansión de cultivos, prácticas agricolas y forestales no sostenibles, crecimiento de zonas urbanas, entre otras, que influyen negativamente tanto en el bienestar de millones de personas como en el de los ecosistemas y las especies que los conforman. La inversión en mecanismos que contribuyan a evitar, reducir y revertir la degradación de la tierra tales como la restauración, traen consigo beneficios que se ven reflejados a nivel económico, social y ambiental en aspectos tan fundamentales como la seguridad alimentaria y de recursos hidricos, mitigación del cambio climático e incluso la prevención de conflictos o migraciones.
\end{abstract}

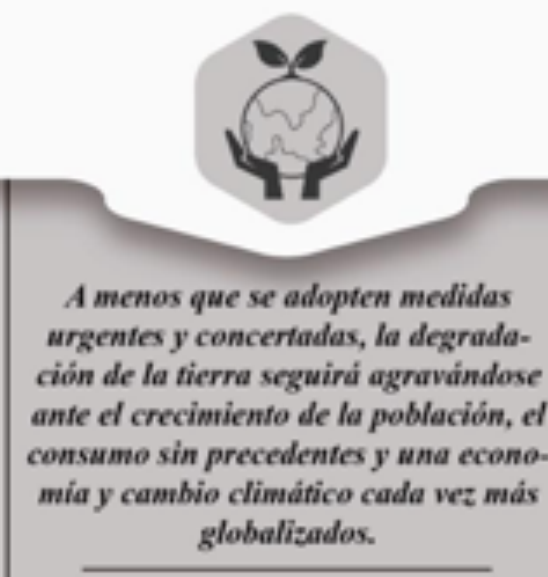

El desarrollo económico, acompañado por un constante aumento en la población y por tanto la desmedida cultura de consumo, se ha convertido en una de las causas más influyentes en el aumento de la degradación, que a su vez contribuye al cambio climático, agravando aún más los efectos de este fenómeno. Las respuestas por parte de los sectores institucionales, politicos y gubernamentales frente a estas problemáticas no resultan ser las más adecuadas, puesto que solo se centran en una de las múltiples causas, esto como resultado del desconocimiento de la existencia de diversos factores impulsores $y$ consecuencias de la degradación de la tierra, sumado a la desvinculación con los demás actores regionales y locales.

Figura 5. Mensajes principales de la Evaluación sobre la degradación y restauración de la tierra Fuente: tomado de la Decisión IPBES 6/15 (2018). 


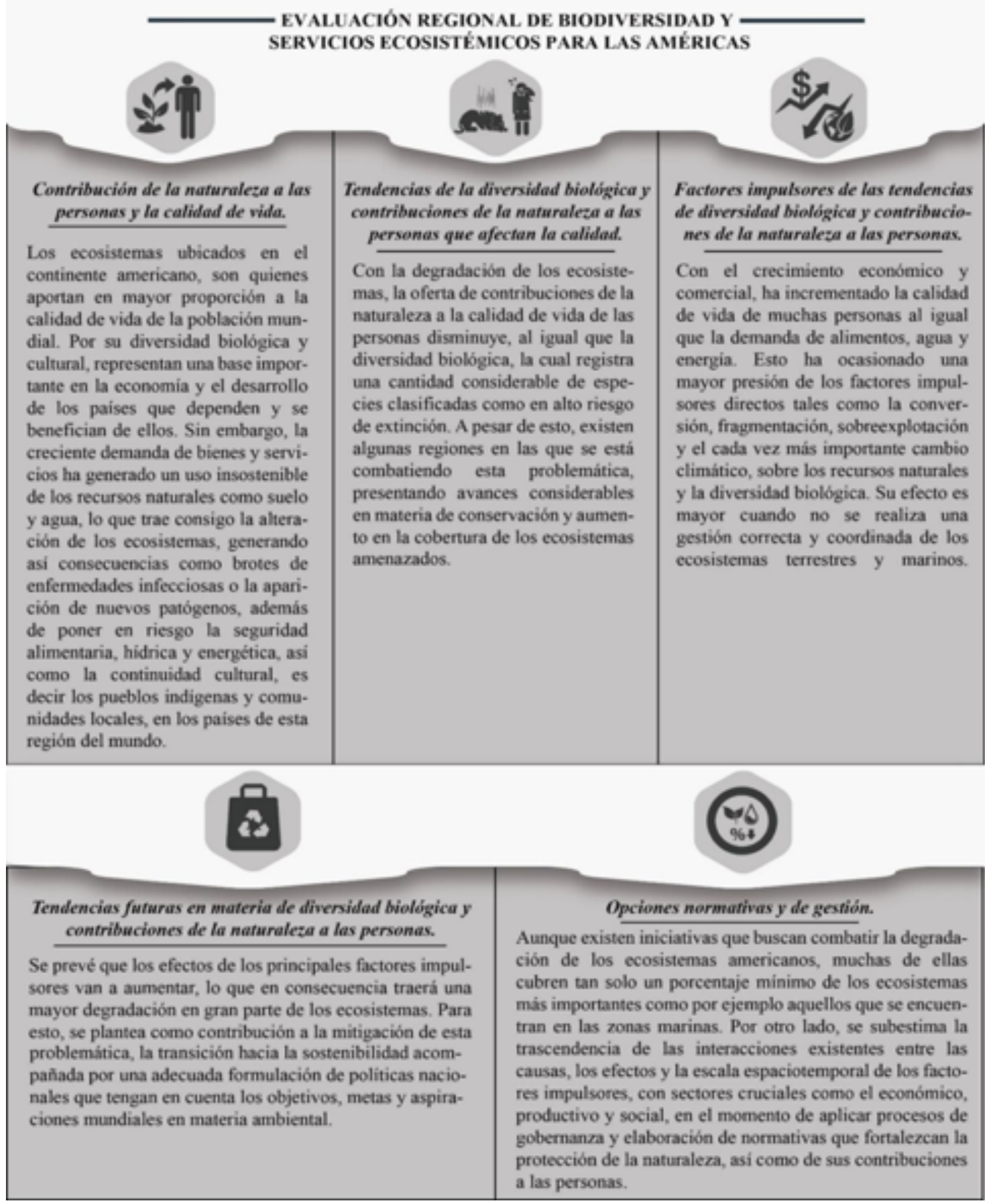

Figura 6. Mensajes principales de la Evaluación regional de biodiversidad y servicios ecosistémicos para las Américas Fuente: tomado de la Decisión IPBES 6/1 (2018). 


\section{EVALUACIÓN GLOBAL DE DIVERSIDAD Y SERVICIOS ECOSISTÉMICOS}

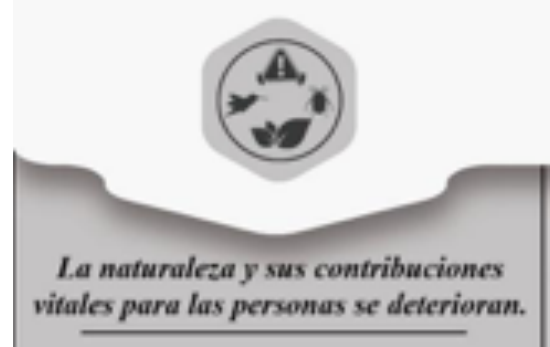

Aunque la naturaleza sea parte esencial para la existencia de las personas, las acciones realizadas por el hombre han afectado severamente stu estructura y funciones, repercutiendo no solo en las contribuciones que brindan los ecosistemas al bombre, que cada vez son menores y distribuidas de manera desigual, sino también en las comunidades biológicas, las cuales con el paso del tiempo, se están volviendo cada vez más similares entre si y están experimentando procesos de evolución acelerados; planteando un panorama en el que se desconoce si las consecuencias de estos cambios serain positivas o negativas para los ecosistemas.

\section{SEVICIOS ECOSISTEMICOS}




\section{DESPUÉS DE LAS EVALUACIONES}

Luego de comprender qué es la IPBES, es necesario advertir la importancia de la cantidad y gravedad de la información que este órgano científico-político ha logrado compilar desde su creación, así como dimensionar su utilidad frente a un cambio real a nivel regional y local. A continuación, se mencionan solo algunas ideas para su uso y potencialización; sin embargo, el único límite de aplicación de este conocimiento es la voluntad política del tomador de decisiones que quiera usarlo y la capacidad de los movimientos ciudadanos para ejercer presión, acción y cambio.

Tabla 1. Reflexiones a partir de las Evaluaciones

\begin{tabular}{|c|c|c|c|}
\hline Evaluación & ¿Es usted gobernante local o regional? & $\begin{array}{l}\text { ¿Es usted científico o investigador en } \\
\text { biodiversidad? }\end{array}$ & $\begin{array}{c}\text { ¿Es usted líder comunitario o pertenece } \\
\text { a algún tipo de ONG? }\end{array}$ \\
\hline $\begin{array}{c}\text { Polinizadores y } \\
\text { polinización para la } \\
\text { producción de alimentos }\end{array}$ & $\begin{array}{l}\text { - Desincentivar el uso de agroquímicos } \\
\text { letales y subletales. } \\
\text { - Proponer incentivos económicos a la } \\
\text { apicultura y sus productos derivados. }\end{array}$ & $\begin{array}{l}\text { - Proponer estudios basados en } \\
\text { polinizadores no carismáticos. } \\
\text { - Buscar vacíos de conocimiento que } \\
\text { puedan ser estudiados, al menos, de } \\
\text { manera local. } \\
\text { - Aumentar la escala espacial de los } \\
\text { estudios de polinización. }\end{array}$ & $\begin{array}{c}\text { - Generar apropiación social del } \\
\text { conocimiento respecto a polinizadores } \\
\text { no carismáticos. } \\
\text { - Proponer proyectos comunitarios } \\
\text { que busquen el sostenimiento de los } \\
\text { polinizadores urbanos. }\end{array}$ \\
\hline Escenarios y modelos & $\begin{array}{l}\text { - Trabajar de la mano con los científicos } \\
\text { que investigan en su jurisdicción. } \\
\text { - Formular políticas basadas en la } \\
\text { información que los científicos brindan. }\end{array}$ & $\begin{array}{c}\text { - Comunicar a los gobernantes locales y } \\
\text { regionales, de manera asertiva y sencilla, } \\
\text { los hallazgos de sus modelos de cambio } \\
\text { elaborados. }\end{array}$ & $\begin{array}{l}\text { - Exigir a la comunidad científica de la } \\
\text { región la divulgación y apropiación del } \\
\text { conocimiento que se está generando. }\end{array}$ \\
\hline $\begin{array}{l}\text { Degradación y } \\
\text { restauración de la tierra }\end{array}$ & $\begin{array}{l}\text { - Modificar su accionar reactivo a } \\
\text { proactivo, tomando decisiones basadas } \\
\text { en la inversión preventiva. }\end{array}$ & $\begin{array}{l}\text { - Trascender los muros de las instituciones } \\
\text { de investigación para trabajar de manera } \\
\text { conjunta con los tomadores de decisiones } \\
\text { en proyectos de prevención, mitigación, } \\
\text { corrección y compensación. }\end{array}$ & $\begin{array}{l}\text { - Concientizar sobre hábitos de consumo } \\
\text { depredadores. } \\
\text { - Educar sobre el impacto real de la } \\
\text { globalización del consumo. }\end{array}$ \\
\hline $\begin{array}{l}\text { Evaluación regional para } \\
\text { las Américas }\end{array}$ & $\begin{array}{l}\text { - Incluir la información de base de la } \\
\text { evaluación en los POT, POMCA o demás } \\
\text { instrumentos de planeación territorial. } \\
\text { - Proponer incentivos económicos a } \\
\text { las empresas y emprendimientos que } \\
\text { usen de manera sostenible los recursos } \\
\text { biológicos y servicios ecosistémicos. } \\
\text { - Incentivar la transición del sector } \\
\text { productivo al uso de energías limpias. }\end{array}$ & $\begin{array}{c}\text { - Procurar la generación de conocimiento } \\
\text { en todos los capítulos que tienen vacíos. } \\
\text { - Buscar la inclusión del conocimiento } \\
\text { tradicional e indígena en las } \\
\text { investigaciones científicas, evitando } \\
\text { a toda costa el colonialismo científico } \\
\text { y procurando una sinergia en ambos } \\
\text { sistemas acerca de la comprensión de la } \\
\text { naturaleza. }\end{array}$ & $\begin{array}{l}\text { - Concientizar a la sociedad sobre el } \\
\text { papel fundamental de Latinoamérica } \\
\text { como despensa alimentaria del mundo. } \\
\text { - Generar un cambio de concepción } \\
\text { sobre el origen de los alimentos y } \\
\text { el agua que consume la sociedad, } \\
\text { realzando el lugar privilegiado que tiene } \\
\text { Latinoamérica en la cantidad y calidad } \\
\text { de producción de los mismos. }\end{array}$ \\
\hline
\end{tabular}

Fuente: elaboración propia.

\section{CONCLUSIONES}

La Plataforma Científico-Normativa sobre Biodiversidad y Servicios Ecosistémicos es una instancia internacional fundamental para la conservación de la diversidad biológica. Este organismo facilita la incorporación del conocimiento científico en las decisiones políticas nacionales de los países miembros, haciendo esta información comprensible para que los administradores de recursos naturales formulen leyes, desarrollen normas, planes o programas que permitan frenar la pérdida de especies y sus interacciones, conscientes de las implicaciones económicas que dicho detrimento genera, así como su impacto sobre el bienestar de la población humana. 
El panorama general que están arrojando las Evaluaciones no es alentador y sin duda alerta a los gobiernos del mundo a tomar medidas urgentes al respecto. También se esperan recomendaciones futuras sobre temas como la industria y su relación con la biodiversidad. De manera resumida se puede afirmar que:

- La biodiversidad y los servicios ecosistémicos de los polinizadores están en riesgo por conductas propias de la aceleración de producción de cosechas y el aumento de su calidad mercantil.

- Existen muchos modelos y escenarios sobre el cambio de la diversidad biológica y sus servicios ecosistémicos, aunque estos no son atendidos por los tomadores de decisiones o no existe suficiente inversión económica para adaptar las políticas a sus resultados.

- La tierra se está degradando y frenar este fenómeno será cada vez más costoso y difícil, sus consecuencias se incrementarán con el cambio climático.

- El continente americano ha puesto en riesgo la biodiversidad y sus servicios ecosistémicos con la implementación de economías extractivistas y de producción intensiva. A este problema se suma el desconocimiento del estado actual de la diversidad de muchas especies y tipos de ecosistemas.

- Los últimos 50 años han sido fatales para todos los ecosistemas, pues estos han sido seriamente afectados por cuenta de la demanda energética generada, a su vez, por el crecimiento poblacional, comercial y económico.

Las opciones con que cuentan los diferentes sectores de la sociedad para poner en práctica las negociaciones realizadas son infinitas y realmente dependen de la voluntad individual y política de cada uno de ellos. Los gobiernos ya no tienen manera de decir que los estudios científicos sobre biodiversidad no son aceptados oficialmente, puesto que las Evaluaciones (basadas en dichos estudios) son jurídicamente vinculantes; es decir, los estados pueden y deben incluir de alguna forma sus contenidos en las políticas nacionales.

\section{AGRADECIMIENTOS}

A Young Ecosystem Services Specialits Network (YESS), orgulloso miembro del Ecosystem Services Partnership (ESP), por el aval de representación ante la IPBES 6 y 7. A Ranjini Murali y Hyeonju Ryu por el apoyo a la delegación en París. Al Semillero G.A.I.A. por su incansable labor de investigación formativa en la última década.

\section{REFERENCIAS}

Álvarez-Dávila, E., Ajiaco, R. E., Buitrago, L., González, M., Laverde, O., Ortiz-Gallego, R., Plata, C., Rosselli, L., Sanjuan, T., \& Uribe, S. (2019). Evaluación nacional de biodiversidad y servicios ecosistémicos. Ministerio de Medio Ambiente y Desarrollo Sostenible, Ministerio de Ciencia, Tecnología e Innovación, Instituto de Investigación de Recursos Biológicos Alexander von Humboldt. http:// www.humboldt.org.co/images/documentos/pdf/ evaluacin-nacional-de-biodiversidad-y-serviciosecosistmicos-borrador-1.pdf

Braat, L. C. (2018). Five reasons why the Science publication "Assessing nature's contributions to people" (Diaz et al., 2018) would not have been accepted in Ecosystem Services. Ecosystem Services, 30(A), A1-A2. https://doi.org/10.1016/j. ecoser.2018.02.002

Brooks, T. M., Lamoreux, J. F., \& Soberón, J. (2014). IPBES $\neq$ I IPCC. Science \& Society, 29(10), 543-545. https://doi.org/10.1016/j.tree.2014.08.004

Convención Sobre la Diversidad Biológica [CDB]. (2014). Memorandum of Cooperation between The Secretariat of the Convention on Biological Diversity and The Secretariat of the Intergovernmental Science-Policy Platform on Biodiversity and Ecosystem Services. IPBES. https:// www.ipbes.net/system/tdf/agmt-ipbes-2014-1009-mou-en.pdf?file=1\&type=node\&id=15239

Convención Sobre la Diversidad Biológica [CDB] (s.f.). SBSTTA Recommendations. Convention on Biological Diversity. https://www.cbd.int/ recommendations/default.shtml 
Convención sobre la Diversidad Biológica [CDB] (2018). Decisión adoptada por la Conferencia de las Partes en el Convenio Sobre la Diversidad Biológica. CDB. https://www.cbd.int/doc/decisions/cop-14/cop14-dec-02-es.docx

Díaz, S., Demissew, S., Carabias, J., Joly, C., Lonsdale, M., Ash, N., ... Zlatanova. D. (2015). The IPBES Conceptual Framework - Connecting nature and people. Current Opinion in Environmental Sustainability, 14, 1-16. https://doi.org/10.1016/j. cosust.2014.11.002

Díaz, S., Pascual, U., Stenseke, M., Martín-López, B., Watson, R. T., Molnár, Z., ... Shirayama, Y. (2018). Assessing nature's contributions to people. Science, 359, 270-272. https://doi.org/10.1126/ science.aap 8826

Faith, D. P. (2018). Avoiding paradigm drifts in IPBES: Reconciling "nature's contributions to people," biodiversity, and ecosystem services. Ecology and Society, 23(2), 40. https://doi.org/10.5751/ES10195-230240

Jonsson, B. G., Báldi, A., \& Lundquist, C. (2017). The Intergovernmental Science Policy Platform on Biodiversity and Ecosystem Services (IPBES). Aglobal model for biodiversity conservation. Reference Module in Earth Systems and Environmental Sciences. http://dx.doi.org/10.1016/B978-0-12409548-9.09766-9

Kenter, J. O. (2018). IPBES: Don't throw out the baby whilst keeping the bathwater; Put people's values central, not nature's contributions. Ecosystem Services, 33, 40-43. https://doi.org/10.1016/j. ecoser.2018.08.002

Laikre, L., Jonsson, B. G., Ihse, M., Marissink, M., Dock-Gustavsson, A. M., Ebenhard, ... Wramner, P. (2008). Wanted scientists in the CBD process. Conservation Biology, 22(4), 814-815. https://doi. org/10.1111/j.1523-1739.2008.00991.x

Organización de las Naciones Unidas [ONU] (1992). Convenio Sobre la Diversidad Biológica. ONU. https://www.cbd.int/doc/legal/cbd-es.pdf

Organización de las Naciones Unidas [ONU] (2013a). Decisión IPBES 2/4: Marco conceptual de la Plataforma Intergubernamental Científico- normativa sobre Diversidad Biológica y Servicios de los Ecosistemas. ONu. https://ipbes.net/ system/tdf/downloads/Decision_2_4_es_0. pdf?file=1\&type $=$ node \&id $=14651$

Organización de las Naciones Unidas [ONU] (2013b). Decisión IPBES 2/5: Programa de trabajo para el período 2014-2018. ONU. https://ipbes.net/ system/tdf/downloads/Decision_2_5_es_0. pdf?file $=1 \&$ type $=$ node $\&$ id $=14657$

Organización de las Naciones Unidas [ONU] (2015). Objetivos de desarrollo sostenible. ONU. https://www.un.org/sustainabledevelopment/ es/2015/09/la-asamblea-general-adopta-laagenda-2030-para-el-desarrollo-sostenible/

Organización de las Naciones Unidas [ONU]. (2018a). Resumen para los responsables de la formulación de políticas del informe de evaluación regional sobre diversidad biológica y servicios de los ecosistemas para las Américas de la Plataforma Intergubernamental Científico-normativa sobre diversidad biológica y servicios de los ecosistemas. ONU. https://ipbes.net/system/ tdf/ipbes_6_15_add.2_spm_americas_spanish. pdf?file $=1 \&$ type $=$ node $\&$ id $=28521$

Organización de las Naciones Unidas [ONU]. (2018b). Resumen para los responsables de la formulación de políticas del informe sobre la evaluación temática de la degradación y la restauración de la tierra de la Plataforma Intergubernamental Científico-normativa sobre Diversidad Biológica y Servicios de los Ecosistemas. https://ipbes.net/ system/tdf/ipbes-6-15-add.5_spm_Idr_spanish. docx ?file=1\&type=node\&id $=28891$.

Organización de las Naciones Unidas [ONU]. (2019a). Decisión IPBES 1/12: Informe del primer período de sesiones del Plenario de la Plataforma Intergubernamental Científiconormativa sobre Diversidad Biológica y Servicios de los Ecosistemas (Anexo II). ONU. https://ipbes. net/system/tdf/downloads/IPBES_1_12_Es_0. pdf?file=1\&type=node $\&$ id $=12369$

Organización de las Naciones Unidas [ONU] (2019b). Summaryforpolicymakers of the globalassessment report on biodiversity and ecosystem services of the Intergovernmental Science-Policy Platform on Biodiversity and Ecosystem Services. ONU. 
Pascual, U., Balvanera, P., Díaz, S., Pataki, G., Roth, E., Stenseke, M., ... Yagi, N. (2017). Valuing nature's contributions to people: The IPBES approach. Current Opinion in Environmental Sustainability, 26(27), 7-16. https://doi.org/10.1016/j. cosust.2016.12.006

Peterson, G. D., Harmáčková, Z. V., Meacham, M., Queiroz, C., Jiménez-Aceituno, A., Kuiper, J. J., ... Bennett, E. M. (2018). Welcoming different perspectives in IPBES: "Nature's contributions to people" and "Ecosystem services". Ecology and Society, 23(1), 39. https://doi.org/10.5751/ES10134-230139

Plataforma Intergubernamental Científico-Normativa sobre Diversidad Biológica y Servicios de los Ecosistemas [IPBES]. (2016a). Resumen para los responsables de formular políticas del informe de evaluación de la Plataforma Intergubernamental Científico-normativa sobre Diversidad Biológica y Servicios de los Ecosistemas sobre polinizadores, polinización y producción de alimentos. IPBES. https://ipbes.net/system/tdf/downloads/ pdf/ipbes_4_19_annex_ii_spm_pollination_ es.pdf?file=1\&type=node $\&$ id $=28364$
Plataforma Intergubernamental Científico-Normativa sobre Diversidad Biológica y Servicios de los Ecosistemas [IPBES]. (2016b). Resumen para los responsables de formular políticas del informe de evaluación de la evaluación metodológica de hipótesis y modelos de la diversidad biológica $y$ los servicios de los ecosistemas. IPBES. https://ipbes.net/system/tdf/downloads/ pdf/ipbes_4_19_annex_iv_spm_scenarios_ es. pdf?file $=1 \&$ type $=$ node $\&$ id $=28347$

Plataforma Intergubernamental Científico-Normativa sobre Diversidad Biológica y Servicios de los Ecosistemas [IPBES]. (2019). Comunicado de prensa: Las contribuciones de la diversidad biológica y la naturaleza continúan en peligroso declive, alertan los científicos. IPBES. https:// www.ipbes.net/news/comunicado-de-prensa-lascontribuciones-de-la-diversidad-biol\%C3\%B3gicay-la-naturaleza-contin\%C3\%BAan- 